\title{
Norois
}

Environnement, aménagement, société

$222 \mid 2012$

Xynthia

\section{Droit de propriété et maîtrise des « sols environnementaux ». Quelques enseignements tirés de la tempête Xynthia}

Property law and the administration of environmental land. Some lessons learned from Storm Xynthia

\section{Pierre-Yannick Legal}

\section{OpenEdition \\ Journals}

Édition électronique

URL : https://journals.openedition.org/norois/4009

DOI : $10.4000 /$ norois. 4009

ISBN : 978-2-7535-1843-8

ISSN : $1760-8546$

Éditeur

Presses universitaires de Rennes

\section{Édition imprimée}

Date de publication : 28 février 2012

Pagination : 79-89

ISBN : 978-2-7535-1815-5

ISSN : 0029-182X

Référence électronique

Pierre-Yannick Legal, « Droit de propriété et maîtrise des « sols environnementaux ». Quelques enseignements tirés de la tempête Xynthia », Norois [En ligne], 222 | 2012, mis en ligne le 30 mars 2014, consulté le 14 janvier 2022. URL : http://journals.openedition.org/norois/4009 ; DOI : https:// doi.org/10.4000/norois.4009 


\title{
Droit de propriété et maîtrise des « sols environnementaux » Quelques enseignements tirés de la tempête Xynthia
}

\author{
Property law and the administration of environmental land \\ Some lessons learned from Storm Xynthia
}

\author{
Pierre-Yannick LEGAL
}

UMR 3128 CNRS, Laboratoire Droit et Changement Social, Université de Nantes, Faculté de droit et des sciences politiques, Chemin de La Censive-du-Tertre, BP 81307, 44313 Nantes Cedex 3, France. Tel. : 33 (0)2 40141576

Fax : 33(0)240141595 (pierre-yannick.legal@univ-nantes.fr)

Résumé : Lors de catastrophes naturelles dévastant des lieux habités, il est d’usage de regarder, une fois les événements passés, la manière dont les lieux avaient été occupés. Très souvent est posée la question du bien fondé de l'installation humaine en de tels endroits, y compris pour rechercher la responsabilité de ceux ayant accordé une autorisation de lotir. Une telle démarche fondée sur le respect du droit de l'urbanisme est souvent oublieuse des sujétions qu'impose le droit de propriété, du moins tel que conçu par le Code civil français. Il est ici proposé de présenter les principales caractéristiques de ce droit et de proposer quelques solutions pour parvenir à réguler les marchés fonciers dans les secteurs sensibles et vulnérables des communes littorales et rétro-littorales. Ces propositions passent par la création d'une catégorie : les "sols environnementaux », que permet le droit de l'environnement, offrant à l'État une maîtrise des sols recouvrée et aux collectivités un contrôle de la rente foncière.

\begin{abstract}
When natural disasters devastate inhabited areas, it is common afterwards at the way the area was occupied. The question of the suitability of human settlement in such places is often raised, including for seeking the responsibility of those who granted the permission to subdivide the land. Such an approach, based on the respect of urban law, is often oblivious to the constraints imposed by property law, at least as conceived by the French Civil Code. What is proposed here is a presentation of the main features of this law and some solutions for achieving a regulation of land markets in the sensitive and vulnerable township of coastal and retro-coastal areas. These proposals involve the creation of a specific category;" environmental soils", which is permitted by environmental law, giving the State control over the soils and the authorities control of land rents.
\end{abstract}

Mots clé : biens immeubles - développement durable - domanialisation - droit de propriété - risques - « sols environnementaux » - souveraineté - rente foncière - zones sensibles - zones vulnérables

Keywords : immovable property - sustainable development - property law - hazards - "environmental soils" - sovereignty - land rent - sensitive areas - vulnerable areas 


\section{INTRODUCTION}

\section{Non jus contra jure ${ }^{1}$}

Chaque catastrophe naturelle fait l'effet d'un révélateur. Dans un monde où l'image joue un rôle prépondérant, où rien de ce qui se déroule en un point quelconque de la planète ne peut être ignoré, mais aussi dans un monde où ce qui échappe au contrôle de l'homme devient nécessairement un sujet sensible, fondé sur l'inquiétude voire l'angoisse, toute manifestation de la nature, une "nature incontrôlable », devient un sujet de préoccupation grave. La machine médiatique s'emballe. Sans discernement, les avis des uns et des autres s'entrecroisent. Aux images télévisées s'ajoutent celles qui circulent sur la toile. Les commentaires vont bon train dans le babillage des «blogs ». L'accessoire prend alors le pas sur le principal. Chacun entend son point de vue et souhaite le transmettre à la terre entière dans une ambiance où se mêlent toutes les formes d'affects. Puis la banalité revient, assez rapidement, avec ce qu'elle peut comporter de médiocre et trop souvent de désolant. L'observateur un tant soi peu éclairé peut enfin reprendre une juste distance en songeant aux vertus de la rationalité (Mercier et Acerra, 2011).

La tempête Xynthia qui a endeuillé maintes familles et a frappé plus particulièrement les départements de la Vendée et de la Charente-Maritime, relève de ces catastrophes naturelles qui suscitent émotions, jeux de solidarités, puis, en un second temps, appellent une somme de réflexions. Il est alors temps de relire les auteurs plus anciens qui se sont exprimés sur l'urbanisation des contrées touchées, de consulter les cartes anciennes et celles établies récemment grâce à des moyens de mesures de plus en plus précis. Il est également recommandé de prêter attention aux transformations humaines qui ont été apportées aux espaces malmenés par les éléments naturels.

Dans le champ des réflexions qui immédiatement surgissent, le droit de l'urbanisme occupe une place centrale. Il est censé, à titre principal, régler harmonieusement et sagement l'utilisation des sols. Si une telle lecture se conçoit, elle mérite, à tout le moins, d'être confrontée au régime juridique d'appropria-

1. Il n'y a pas de loi contre le droit. tion des sols. Un tel exercice permet de mesurer ce que recouvre aujourd'hui la notion complexe, et partant confuse, de droit de propriété privée. Pour revenir au cas d'espèce, les parcelles bâties submergées avaient été assez récemment organisées en forme de lotissements, acquises par des particuliers qui y avaient fait édifier des maisons individuelles à usage d'habitation ${ }^{2}$. Ils en étaient propriétaires et jouissaient de toutes les prérogatives que le statut civiliste arrête, statut protégé par des normes à valeur constitutionnelle, reconnues par le droit français et européen ${ }^{3}$.

Les territoires visés par le présent article concernent les ensembles communaux littoraux et rétro-littoraux, notamment ceux de la façade de l'Ouest Atlantique, mais nombre d'observations générales sont de nature à s'appliquer à d'autres espaces (Renard, 2008). Partons d'un constat local. L'appropriation des terrains proches des rivages de la mer s'explique par un certain nombre de paramètres : les lieux sont agréables, prisés; il y fait bon vivre et le marché foncier a permis à plusieurs strates de la société, plus ou moins aisées, d'accéder à la propriété, et partant de réaliser un rêve. Les statistiques d'occupation des côtes vendéennes offrent une vision claire des réponses apportées depuis les soixante dernières années pour rendre compte de ce que la pression foncière signifie (Hussenet, 2004; Legal, 2008; Renard, 2008). Dans ce laps de temps, toutes les communes littorales ont été loties, et la couverture bâtie se poursuit dans l'aire communale rétro-littorale. Certes, des lieux épargnés par cette urbanisation demeurent. Ils jouent le rôle de coupures vertes. Ils sont constitués de propriétés domaniales, essentiellement sous forme de forêts littorales, et de terres vouées à l'agriculture. Les prévisions montrent que la tendance de densification des constructions se poursuivra avec quelques inflexions liées à une situation économique moins favorable, à la gestion du stock des biens immobiliers existants acquis par une génération vieillissante

2. Une étude complémentaire devrait être conduite pour les terres submergées à usage agricole. Pour autant, un certain nombre d'observations générales visent également ces espaces qui ont été envahis par les eaux saumâtres.

3. Articles 2 et 17 de la Déclaration des Droits de l'Homme de 1789 , inclus dans le bloc de constitutionnalité complétant les dispositions de la Constitution du $1^{\text {er }}$ septembre 1958 et l'article $1^{\text {er }}$ du protocole $n^{\circ} 1$ à la Convention européenne de sauvegarde des droits de l'homme et des libertés fondamentales. 
et des politiques de protection des milieux naturels (Hussenet, 2008).

La volonté opiniâtre de s'établir en bord de mer ou à proximité met à mal quelques règles de prudence, notamment celles qui ont pour objet de veiller au respect des zones sensibles. Cette expression englobe les secteurs particulièrement dangereux, fortement exposés aux éléments (vent, eau de mer, etc.) et aux submersions par des jeux complexes où se mêlent les effets des dynamiques marines et fluviales ${ }^{4}$. La prudence renvoie également à la protection de lieux-dits vulnérables, zones originales et fragiles, qui méritent d'être conservées soit comme éléments caractéristiques d'un paysage, soit, et parfois en même temps, pour leur intérêt floristique et faunistique... De tels espaces peuvent être urbanisés ou utilisés à des fins agricoles ou conchylicoles à l'expresse condition de mener une politique de contrôle des sols conçue en toute clarté et rigueur. En revanche, il n'est pas concevable d'y laisser se développer une activité humaine non régulée, à peine de constater qu'en peu de temps des installations individuelles autorisées ou implantées en marge de la loi encombreront le paysage.

La détermination de ces zones correspond à des définitions juridiques incluses dans le Code de l'urbanisme et le Code de l'environnement. Il est relativement simple, dans le cadre de la mise en œuvre d'une politique volontaire, d'en arrêter les contours. Chacun sait les progrès de la cartographie susceptible de prendre en compte les zones submersibles (Robin, 2002). En outre, dans chaque département, une somme de connaissances répertoriées est aisément mobilisable. Elles sont le fruit de travaux d'historiens, de géographes, d'urbanistes, d'érudits, de militants associatifs, de personnels des administrations d'État, départementales et communales, etc. La constitution d'atlas, en usant de fichiers informatiques, relève d'une simplicité technique à la condition qu'il y ait un maître d'œuvre patenté. En outre, grâce à de nombreux observatoires, et donc à des observateurs reconnus, ces cartes peuvent être corrigées très rapidement à partir de mises à jour que les outils informatiques actuels autorisent. Ainsi dans chaque département mais aussi, pour trouver

4. Ces dynamiques concernent les estuaires et les fonds de rias. Ainsi en Vendée les estuaires de la Vie à Saint-Gilles-Croix-de-Vie, de l'Auzance au Havre de la Gachère, du Lay entre les communes de La Faute-sur-Mer et l'Aiguillon-sur-Mer. des aires pertinentes, à l'échelle de toute région administrative, la centralisation des informations sur toutes zones sensible ou vulnérable devrait être conduite rapidement en vue d'établir un «tableau de bord » immédiatement consultable par l'administration préfectorale et toute personne en charge de responsabilité. Dans un pays comme la France, un tel dossier relève tout naturellement du rôle traditionnellement assigné à l'État et à ses principaux administrateurs.

Toutefois, parmi les difficultés à vaincre pour parvenir à des résultats tangibles dans le cadre d'une politique d'urbanisation maîtrisée, il convient de mesurer la force de la notion de droit de propriété privée dont on sait le caractère sensible. La démarche proposée entend partir de l'appropriation privée envisagée sous le double angle des qualificatifs techniques fondés sur les caractères exclusifs et absolus qu'assigne l'article 544 du Code civil. Certes ces termes, arrêtés en 1804 dans un contexte historique et économique particulier, renvoient à des concepts évolutifs mais qu'il faut se garder de considérer comme désuets, de nature à être remisés au magasin des accessoires. La construction du droit de propriété suit des évolutions aisément discernables. Reste qu'elle s'édifie à partir, et parfois autour, du socle que représente toujours le Code civil. En outre, la sédimentation du droit de propriété perdrait tout sens intelligible sans être reliée à la façon dont les propriétaires conçoivent les modes d'appropriation (Xifaras, 2004). La manière de s'approprier un bien mérite une attention particulière dans les espaces sensibles et vulnérables.

Pour mener à bien cette réflexion, trois étapes sont proposées. La première consiste à analyser la notion de propriété privée et à mesurer l'efficience des techniques de régulation des caractères exclusifs et absolus. La deuxième renvoie à la notion combinée de propriété, de prise en compte des risques et de la rente foncière, questions qui en droit privé français est largement laissée pour compte. Enfin, la troisième consiste à présenter quelques moyens de protéger efficacement certains espaces, y compris contre la marche implacable des marchés.

\section{Réguler le droit de PRopriété}

Le droit de propriété s'exerce sur un bien. Dans la panoplie des catégories classiques structurant 
le droit français, issues d'un droit romanisé au $\mathrm{XVI}^{\mathrm{e}}$ siècle, les biens sont meubles ou immeubles, selon l'énoncé du Code civil en son article 516 rédigé en forme principielle. Notre propos délaissera les biens meubles et visera tout spécialement les biens immeubles, savoir les fonds de terre, et par incorporation, les constructions édifiées sur ces parcelles. Elles deviennent, à l'instar des arbres et autres plantes enracinées dans le sol, des biens immeubles, suivant ainsi un régime spécifique et protecteur que commandent les articles 517 à 526 du Code civil.

Le titulaire d'un droit de propriété exerce sur le bien immobilier un lien direct qui lui confère des prérogatives exclusives et absolues. Ces termes sont entendus dans une acception juridique stricte (Zenati-Castaing et Revet, 2008). Ils emportent nécessairement des conséquences immédiates, bien qu'évolutives, que la doctrine souligne avec constance depuis des décennies (Terré et Simler, 2006). Le propriétaire dispose d'un ensemble d'actions en vue de défendre son bien (ses droits) contre les menaces du voisinage, à l'encontre des activités nocives de tiers. Il jouit également de toute latitude pour agir envers ceux qui entendent réduire ses prérogatives d'user et de disposer de son bien. Il exerce un imperium sur un bien qui se conçoit comme un lien direct entre le titulaire du droit et la chose, lien qui, par raccourci, s'entend de préférence entre une personne juridique et une propriété. Dans l'esprit des fondateurs du droit privé, une telle conception repousse vers des régimes spéciaux, et donc fragilisés, toutes formes de propriétés partagées, comme la copropriété des immeubles bâtis ou l'indivision.

Fondé sur le Code civil, d'essence législative, le droit de propriété, ainsi radicalisé, obéit à une logique constitutionnelle. Toute minoration ou altération des prérogatives dudit droit est soumise au vote d'une loi en vertu de l'article 34 de la Constitution du 4 octobre 1958. En outre, ladite loi doit être conforme à un ensemble de normes supérieures françaises et européennes protégeant le propriétaire, lequel peut à tout moment exciper du statut de droit naturel qui entourent le droit de propriété, ouvrant immédiatement des protections imprescriptibles 5 . Autant souligner que les règles

5. La question préalable de constitutionalité (QPC) ouvre aujourd'hui des voies nouvelles de contestation de la loi devant le Conseil constitutionnel dont on ne mesure pas encore complètement l'étendue alors que ce qui fondent le droit de propriété évoluent selon des parcours qui ne suivent pas les diagonales que la doctrine voudrait parfois voir adopter. De longs méandres, en raison des jeux de pression, caractérisent les modes de socialisation d'un droit qui englobe des segments entiers de l'économie.

Dans une telle conception, le propriétaire possède des prérogatives étendues, diminuées par deux types de restrictions acceptables. Les premières sont tirées du droit de propriété lui-même. Lois et jurisprudence sanctionnent les attitudes de propriétaires qui agissent dans une forme jusqu'au-boutiste de leur droit. La Cour de cassation a depuis fort longtemps, s'appuyant sur une partie de la doctrine, établi la notion d'abus de droit (Patault, 1989; Bergel, 2002). Dans un domaine tout autre, la jurisprudence, en accord avec une doctrine surabondante, sanctionne les tentatives de restrictions des prérogatives des propriétaires menées par la puissance publique. À défaut de règles correctement établies, répondant strictement à la notion d'utilité publique, les limitations aux droits des propriétaires, mal fondées, sont sanctionnées par les juridictions administratives, dont le Conseil d'État, sans préjudice d'autres instances juridictionnelles, françaises ou européennes, comme la Cour de cassation et la Cour européenne des droits de l'homme. Ainsi, toutes limitations tendant à contrecarrer le droit de disposer (abusus), de jouir (fructus), et d'utiliser (usus) un bien, composantes essentielles du droit de propriété, seront impitoyablement réduites voire interdites.

Cet exposé sommaire invite à ne pas inverser les perspectives que l'apparence laisse supposer. Chaque fois que la libre cession d'un bien et son utilisation sont en jeu, le droit du propriétaire prime, fondé sur des principes généraux soigneusement gardés. Les règles d'urbanisme et celles qui découlent du droit de l'environnement relèvent de dispositions spéciales subordonnées auxdits principes. Dans la conception française du droit, l'exception est strictement limitée au champ d'application du texte qui l'a créée. En cas d'imprécision, dans le fourmillement de textes d'exception, les principes

Conseil n'a pas été conçu, du moins par rédacteurs de la constitution de 1958, comme une véritable juridiction. La jurisprudence de la Cour européenne des droits de l'homme (CEDH), retient aujourd'hui l'attention de tous les juristes français. Cette juridiction coiffe désormais, d'une certaine manière, la Cour de cassation, et d'ici peu, le Conseil d'État, en ses fonctions contentieuses, dont la notion d'exception à la Française ne sera pas longtemps crédible et est d'ores et déjà contestée. 
généraux bénéficieront au propriétaire auquel une règle mal établie sera réputée inopposable.

À l'issue d'une présentation rapide du droit de propriété conçu comme un dispositif structuré encadré par le Code civil, il importe de s'intéresser à l'objet auquel il s'applique. La parcelle de terre identifiée, cadastrée, élément immobilier par nature, relève de la catégorie des biens patrimoniaux. Elle est évaluable en argent et entre dans une économie de marché. Ce bien se caractérise par sa rareté. En raison de l'accroissement de la population de la planète, augmentée de la pression des demandes en certains secteurs du globe, la terre est convoitée. Nul ne peut ignorer que la libre disposition de toute parcelle est de droit strict, sauf être encadrée par une règle limitative législative au contenu incontestable devant une juridiction. L'usage du même bien notamment en vue de construire un édifice à usage d'habitation ou de commerce obéit aux mêmes sujétions. Ces deux éléments renvoient aux notions classiques d'abusus et d'usus qui forment deux composantes du droit de propriété, augmentées d'une troisième, le fructus, qui ne présente pas un intérêt immédiat dans notre dossier.

En premier lieu, les règles d'urbanisme sont venues tempérer le droit de propriété, voire le contraindre. Elles ont timidement vu le jour dans l'entre-deux-guerres, et ont été affirmées sous l'égide de l'État français, période de forte puissance de l'administration ${ }^{6}$. Les ordonnances de 1944 et 1945 ont repris ces textes qui ont connu dans les décennies suivantes une véritable inflation au point de justifier une codification en 1954, suivie d'une " codification-partage » en 19727. Les lois des 2 mars 1982 et 7 janvier 1983, portant respectivement sur le principe de la décentralisation et la répartition des compétences entre l'État et les collectivités locales, ont modifié sensiblement les modes de décisions dans la maîtrise des sols. D'année en année, tout un arsenal visant à restreindre le droit de propriété privée a eu pour objet de le limiter et d'encadrer le droit de construire (usus) et de disposer de son bien (abusus). Ce droit de l'urbanisme a été complété, voire doublé, par les premières mesures excipant

6. Loi du 15 juin 1943 constituant la première codification raisonnée en matière d'urbanisme.

7. Loi du 30 juin 1972 arrêtant la création d'un Code de la construction et de l'habitation et un Code de l'urbanisme. du droit de l'environnement auquel il est, du moins dans ses premières années, fortement ressemblant.

Le droit de l'urbanisme et de l'environnement ne sont pas conçus à la manière du droit de propriété. Ils ne sont pas de même bois. En effet, le droit de propriété repose sur un ensemble de principes énoncés de manière générale et impersonnelle dans le Code civil et dans quelques textes fondateurs selon un modèle hérité du droit romano-canonique médiéval, rationalisé au cours de la période moderne. Dans le Code civil, rares sont les exceptions aux principes. Quant aux évolutions des concepts, elles sont confiées à la jurisprudence. Cette construction prétorienne est principalement l'apanage de la Cour de cassation. S'agissant du droit de l'urbanisme et du droit de l'environnement, ils s'apparentent davantage à un droit casuistique, porteur de mille touches particulières qu'il est parfois malaisé de relier entre elles. Certes, quelques grandes lois tentent de structurer l'édifice mais la couture ne masque guère un caractère artificiel, et partant fragile ${ }^{8}$. D'un certain point de vue, le droit de l'urbanisme, qui renvoie à la catégorie du droit public, serait plus proche du droit anglo-saxon, avec son caractère pointilliste.

Le droit de l'urbanisme et le droit de l'environnement naissant présentent un caractère de fragilité, voire de subsidiarité, au regard du droit de propriété privée. Appliqués à l'exemple vendéen, ils autorisent trois types de réflexions. En premier lieu, l'application des règles a été peu contraignante. En cinquante ans, ont été construits des lotissements sans âme, et ce dans toutes les communes littorales et rétro-littorales, et des immeubles les pieds dans l'eau comme à Merlin-Plage ou à Saint-Gilles. Le remblai des Sables-d'Olonne a largement été déstructuré et la forêt de La Tranche-sur-Mer mitée ${ }^{9}$. La déprise agricole a permis la création de tout un immobilier de loisir d'assez médiocre qualité. Aujourd'hui, les terrains de camping se couvrent de bungalows en plastique, avatars des cabanes de chantiers, pompeusement dénommés «mobil home », concédés à des particuliers en des contrats qui démembrent le droit de propriété entre le droit de tréfonds et celui de superficie. Comme nombre d'auteurs ont pu le constater, le droit de l'urbanisme a davantage servi

\footnotetext{
8. La loi du 13 décembre 2000, dite loi SRU (Solidarité et renouvellement urbain) tente de répondre à ce besoin qui s'impose au législateur tous les vingt ans...

9. Il ne s'agit là que de quelques exemples classiques. Ils pourraient être bien plus nombreux.
} 
de mode de distribution de droits à construire qu'objet de régulation et de maîtrise du foncier (Chauvet et Renard, 1978).

En second lieu, la complexité du droit de l'urbanisme a permis à nombre de plaideurs de contester le bien fondé des règles établies et à faire triompher leurs prérogatives de propriétaires. Cette branche du droit est un nid à procès qui se règlent essentiellement devant les juridictions administratives réputées non coûteuses pour le citoyen. Les enjeux en valent souvent la peine ${ }^{10}$. Enfin, les textes, même clairs, ont souvent été adoptés de manière différée et ont été mal appliqués. La loi Littoral qui aurait dû être votée à la fin de la décennie soixante, n’a finalement et laborieusement vu le jour qu'en 1986. Nul n'ignore également que son véritable premier décret d'application a été signé en $2004^{11}$. Ceci ne signifie pas que le texte soit resté lettre morte, mais son efficacité a largement été freinée. Les rapports du Sénat et de l'Assemblée nationale en date du 21 juillet 2004 ne manquent pas de sévérité à ce propos $^{12}$. Il n'échappe à aucun observateur que les Plans de prévention des risques naturels prévisibles ne sont toujours pas adoptés dans un certain nombre de communes concernées, en dépit de la pression préfectorale ${ }^{13}$. Des élus tentent de repousser leur mise en œuvre afin de conserver une maîtrise de la constructibilité des terrains vacants, y compris ceux placés à une faible altitude par rapport au niveau des eaux de la mer ou d'une rivière. Avant la tempête Xynthia, les préfets du département de la Vendée ont souvent eu maille à partir avec les maires

10. Du moins si l'on songe aux intérêts immédiats des seuls propriétaires... Nous renvoyons à l'article de P. Le Louarn dans cette livraison. Il montre parfaitement comment les divers droits spéciaux ne s'accommodent pas nécessairement. Leur mise en œuvre se heurte à des interrogations fortes, ce que n'a pas manqué de relever la commission ad hoc ayant rendu son rapport en 2010. En outre, bien des habitués du droit de l'urbanisme littoral ne méconnaissent pas les distorsions existant entre la loi Littoral de 1986 et les dispositions découlant de la loi SRU de 2000.

11. Un premier décret pris le 20 septembre 1989, codifié sous l'article R. 146-1 du Code de l'urbanisme, était redondant avec le texte de loi, imprécis dans le champ d'application du texte et a conduit à nombre de confusions. Les deux décrets attendus et réclamés, y compris par le Conseil d'État, ne sont intervenus qu'en 2004. Il y a peu d'exemples similaires dans notre droit où les décrets d'applications d'un texte fondamental, ont été adoptés avec autant de mauvaise grâce. Rappelons qu'initialement, au pied de la loi, neuf décrets d'application devaient être pris dans un délai raisonnable.

12. Rapport sénatorial établi sous la présidence de P. Gélard, et rapport de l'Assemblée nationale rédigé sous la présidence de J. Le Guen. Ces documents sont consultables sur les sites des deux assemblées. « Le mal est déjà fait », souligne le rapport Le Guen puisque la jurisprudence a pris le pas sur la source réglementaire. Les décisions des juridictions administratives ont apprécié le champ du texte de manière beaucoup trop restrictive.

13. Article L. 562-1 du Code de l'environnement. concernés afin de faire respecter une loi pourtant impérative et adoptée depuis près de quinze années (Bécet, 2002) ${ }^{14}$.

Utiliser le droit de l'urbanisme ou en freiner, le cas échéant, l'application renvoie à la notion de valeur marchande du bien immobilier. Nous avons souligné ci-dessus les remarques de nombreux auteurs sur le rôle joué en ce sens par le droit de l'urbanisme, dispositif de distribution de droits de construire. Mais poussant l'analyse plus avant, il est loisible d'affirmer que l'exercice de ce droit conduit à dispenser la richesse quand le terrain peut être loti. Devenu constructible, il prend immédiatement de la valeur et devient aisément cessible tant la demande est forte. Le goût du lucre prend alors souvent le pas sur les exigences de protection et de précaution. La loi Solidarité Renouvellement Urbain (SRU) a-t-elle changé les modes d'actions en ce domaine. Appliquée depuis dix ans, on ne mesure pas aisément ses incidences quand on observe l'étalement urbain à proximité des côtes. Ce texte, comme bien d'autres avant lui contient les germes de son impuissance (Lebreton, 2009).

Depuis les lois de décentralisation de 1982 et de 1983, l'échelon communal a été privilégié avec un traitement égalitaire entre les communes d'importance dotées de services de conception des plans d'urbanisme et d'instruction des permis de construire, et les petites devant faire face aux tâches multiples d'une complexité sans cesse accrue. Quant aux responsabilités des élus, elles relèvent d'une même nature. Établir un plan d'occupation des sols, hier, ou un plan local d'urbanisme, aujourd'hui, relève d'un exercice que seuls les élus des grandes agglomérations et des villes moyennes peuvent maîtriser avec une juste distance. Dans une petite commune littorale ou rétro-littorale, le maire et ses adjoints sont soumis à une somme de pressions qu'ils ne peuvent raisonnablement mâ̂triser. La proximité des administrés, y compris ceux inclus en des maillages familiaux et d'alliances, ne peut donner lieu à une administration sereine de droits à construire. On objectera à cette remarque que les services de l'État peuvent venir en appui. Mais c'est être oublieux de deux éléments qui

14. Article 16 de la loi du 2 février 1995 relative au renforcement de la protection de l'environnement, modifiant la loi du 22 juillet 1987. Cet article fixe le principe et les modalités d'élaboration des plans de prévention des risques naturels prévisibles. Ce texte a été abrogé et remplacé par l'ordonnance du 18 septembre 2000. 
pèsent lourdement aujourd'hui. En premier lieu, les Directions Départementales de l'Equipement (DDE), dénommée aujourd'hui DDTM ${ }^{15}$, qui assuraient, sous contrôle de l'État, l'instruction des permis de construire ont perdu une bonne partie de leur personnel qualifié en ce domaine. En outre, une même DDTM ne saurait dans le cadre d'une administration sereine, être mandatée par une commune pour instruire un permis de construire à la demande d'un maire, es-qualité de prestataire de service, et jouer le rôle de gardien du droit dans le cadre de l'exercice de pouvoirs régaliens. En second lieu, le Préfet assure sur les permis de construire un contrôle de légalité qui, très souvent, ne peut raisonnablement porter que par sondage. Le droit de la décentralisation a, de ce point de vue, fortement diminué l'efficience des prérogatives préfectorales.

La faiblesse de l'échelon communal se révèle également patent en deux volets aisément observables. D'une part, les communes littorales peinent à faire respecter les règlements qu'elles ont, elles-mêmes, édictés. Dans toutes les communes littorales ou rétro-littorales, des terrains non constructibles ont été acquis par des propriétaires qui y ont installé une caravane, puis un abri de jardin, puis progressivement une construction. La plupart de ces lieux sont édifiés en bordure de zones sensibles, en des espaces classés. Ces établissements devenus pérennes sont dépourvus de dispositifs d'assainissement, parfois d'électricité. Quelques initiatives ont été prises çà et là pour faire respecter la loi. Bien peu de réussites ont pu être constatées en ce sens. Une fois encore, les droits du propriétaire ont prévalu sur les prérogatives des élus, voire même de l'administration. Un parcours dans les marais d'Olonne, lieux d'exception bénéficiant de toutes les protections possibles, laisse entrevoir des constructions pour le moins curieuses et qui mériteraient une étude fine prenant en compte le droit des sols et les règles relatives à la protection des paysages, de la faune et de la flore.

D'autre part, l'incapacité des communes littorales à résister à la pression foncière est évidente tant la demande en terrains à bâtir est forte. Elle se manifeste aujourd'hui par l'édification de maisons sur toute parcelle constructible. Les terrains font l'objet de partages et de divisions foncières et la densifica-

15. Devenue Direction Départementale des Territoires et de la Mer (Vendée). tion est aisément perceptible pour tout observateur un rien attentif. Il n'est peut-être pas mauvais que ces constructions voient le jour à condition toutefois qu'elles répondent à une politique maîtrisée de l'urbanisme. Mais il y a également lieu de penser que la pression n'est guère régulée et que le marché foncier, la filière du bâtiment et l'ensemble des entreprises qui en dépendent, pris dans une spirale, invitent à ne pas ralentir la progression. Une cartographie mise à jour, et tenant compte des normes nouvelles adoptées après la tempête Xynthia, indiquera sous peu combien de parcelles se situent dans une zone à risque, susceptible d'être submergée en cas de montée des eaux ou de subir une destruction en raison de son exposition. La consultation de ces cartes sera, sans aucun doute, instructive.

Pour conclure sur la question de la contrainte du droit de propriété et de la maîtrise des sols, il importe avant tout de retrouver le bon niveau de décisions avant de s'ingénier à légiférer à outrance. La réforme globale du droit de propriété ne sera guère adoptée avant de nombreuses années. Mieux vaut alors actionner des leviers plus simples, destinés à faire appliquer les textes existants afin d'arrêter une politique contrôlée de la consommation des sols dans les communes littorales et rétro-littorales ${ }^{16}$. Une telle politique qui renvoie à la notion de sécurité des personnes et de respect de l'environnement, relève de la compétence de l'État. Il s'agit là d'éléments constitutifs de la souveraineté de l'État (Maulin, 2003). Certes, ce terme est, de nos jours, assez médiocrement connoté par jeu de confusion sémantique. La notion de souveraineté mérite d'être refondée en veillant à ce que certaines prérogatives visant à protéger les personnes et certains biens le soient dans le cadre d'un intérêt général fondé sur l'ancienne notion aristotélicienne d'utilitas devenue par jeu d'imprégnation l'utilité publique.

Ainsi, par ordre du premier ministre, dans chaque département, un inventaire des zones à protéger devrait être dressé dans un délai très court. Les protections pourraient porter sur les zones submersibles, vulnérables, sensibles et remarquables, dont les définitions figurent dans les Codes de l'urbanisme et de l'environnement. Dans lesdites zones

16. Ceci n'exclut pas, bien au contraire, de toiletter certaines dispositions repérées comme empêchant un fonctionnement harmonieux de la politique de maîtrise foncière. On retiendra l'exemple des contradictions entre loi Littoral et mise en œuvre des PLU. 
déterminées, le droit de construire devrait être soumis à un contrôle effectif du préfet de région à qui reviendrait l'obligation de délivrer le permis de construire après avoir reçu un avis du maire de la commune et du préfet du département considéré. Le préfet de région pourrait utilement solliciter divers avis complémentaires dont ceux des présidents du conseil régional et du conseil général territorialement compétents. Dans le cadre de cette procédure d'instruction et de décision, la notion de risque serait nécessairement prise en cause et notifiée au pétitionnaire. Au préalable la confection des Schémas de cohérence territoriale et des Plans locaux d'urbanisme devrait être approuvée par le préfet de région avant de devenir exécutoires.

À l'issue de cette première étape qui nous a conduit à confronter droit de propriété et droit d'urbanisme, il importe d'interroger les questions de propriété liées aux notions de risques et de rente foncière.

\section{Propriété, risques ET RENTE FONCIÈRE}

La pression sur les élus dispensateurs des droits à construire est d'autant plus forte que tout classement en zone constructible confère au propriétaire des parcelles de terrain considérées des droits subjectifs augmentés de droits incorporels accessoires. Les droits subjectifs consistent en la capacité de vendre librement la pièce de terre en question. Les droits corporels accessoires résident en l'autorisation administrative de construire qui découle du classement du terrain en zone aedificandi. Cette autorisation, qui, initialement, relève du droit public, entre ensuite dans le champ du droit privé patrimonial. En effet, elle devient l'accessoire de la parcelle du terrain considéré, en vertu du principe : « L'accessoire suit le principal » (Lévy et Castaldo, 2002) ${ }^{17}$. Elle est créatrice d'une valeur monnayable cessible.

Le classement d'une parcelle de terrain en zone constructible confère immédiatement une plusvalue dans le patrimoine du bénéficiaire sans qu’il ait à effectuer le moindre acte de commission. Dans la tradition juridique française, notre bénéficiaire n'a rien, ou si peu, à verser à la collectivité qui lui a accordé le droit de construire par jeu de décision

17. Principe hérité du droit romain et fortement remis en application par les juristes rationalistes du XVIe siècle : Accessorium sequitur principale. favorable du Plan local d'urbanisme (PLU). À l'inverse, le propriétaire d'une parcelle dont le classement n'ouvre pas aux droits à construire ne peut réclamer aucune indemnité. Il n'existe, lors de la mise en application d'un PLU, et il en allait même avec les plans d'occupation des sols, aucune péréquation entre les propriétaires bénéficiaires, en raison de la situation de leurs propriétés, et ceux moins chanceux. On peut comprendre aisément la force des tensions et les jeux de pressions.

La plus-value immédiatement évaluable lors de l'énoncé de la constructibilité d'un terrain, prend nom de rente foncière. Elle peut être conservée en capital et ne sera liquidée qu'au moment de la cession de la parcelle considérée. Dans un esprit libéral à la française, et en raison des dispositifs juridiques ci-énoncés, cette rente appartient automatiquement et intégralement au propriétaire de la parcelle. Les collectivités publiques n'en bénéficient qu'accessoirement, et de façon différée, à travers la perception de taxes assises sur le prix de la vente de la parcelle et, le cas échéant, par le jeu de la taxation des plusvalues immobilières. Ce calcul n'est établi qu'en cas de vente du bien et non s'il est utilisé par son propriétaire. Ces impositions sont d'ordinaire des plus raisonnables.

Répartir la rente foncière n'est pas une idée nouvelle. Elle a été défendue au début du siècle dernier par M. Halbwacks alors que le sociologue prenait conscience des mutations des valeurs des terrains concernés par les aménagements urbains parisiens (Halbwachs, 1908). Son propos n'avait guère de chances d'être entendu dans une Troisième République qui brilla par son conservatisme dans sa conception du droit de propriété et dont le programme social a été étique. Les préconisations de M. Halbwacks étaient pour autant assez simples, y compris dans leur mise en œuvre. Les municipalités étaient invitées à reprendre une partie substantielle de la plus-value foncière par l'impôt. Cette manne fiscale aurait pu être utilisée pour financer des équipements publics utilisables par tous, et pour conduire une politique sociale notamment dans le domaine du logement. Dans la France du $\mathrm{Xx}^{\mathrm{e}}$ siècle, aucun régime politique n'a pris le risque d'adopter une telle mesure de justice et d'équité. Dans le même laps de temps, il est également observable qu'aucun gouvernement n'a osé réformer la propriété du Code civil, se bornant à adopter diverses 
lois partielles sans pour autant remettre en cause l'architecture napoléonienne. Les invitations de M. Halbwacks demeurent d'une criante actualité. Ce ne sont pas les taxations actuelles de plus-values immobilières qui aujourd'hui, y compris avec un durcissement annoncé du régime, modifieront sensiblement le rapport entre propriété et profit tiré de la rente foncière.

La réflexion autour de la finance mérite d'être poussée sur le terrain du risque. Lorsqu'un propriétaire obtient des droits à construire pour un terrain placé dans une zone à risques, dans une conception libérale à la française, il jouit des lieux en plein propriétaire. Le plan de prévention des risques et quelques autres sujétions urbanistiques particulières le conduisent à réaliser toute construction en respectant un ensemble de prescriptions qui, sans nul doute, alourdissent le coût de la réalisation de son projet. Toutefois, une fois ces exigences satisfaites, il se trouve donc en position de créancier de droits à l'endroit de la commune, du département ou de l'État qui doit assurer la protection extérieure à sa propriété. À la collectivité de supporter le coût de ladite protection par la réalisation d'ouvrages d'arts (digues, enrochements, exutoires d'eau, etc.). En outre, en cas de dommage à sa personne ou à ses biens, la collectivité sera sollicitée pour contribuer aux réparations. Dans l'article figurant dans ce numéro de Norois, P. Le Louarn, à propos des conséquences de la tempête Xynthia, a exposé le mécanisme des transferts de risques, et donc de coûts entre propriétaires, assureurs et citoyens devant contribuer, ouvrant à des modes de solidarité déséquilibrés. Manifestement la rupture d'équité a été consommée.

Deux hypothèses méritent d'être prises en compte. La première concerne le propriétaire d'une parcelle de terrain placée en zone à risques déclarée constructible. Le titulaire du droit de propriété réalise immédiatement une plus-value, sans avoir à supporter de charges. En raison de la notion de risque, la plus-value réalisée à première cession devrait donner lieu à forte taxation au bénéfice d'un fonds destiné à prendre en charge les travaux qui incombent et incomberont à la collectivité devant construire et entretenir tous ouvrages de protection et mettre en place tous services destinés à prodiguer des secours d'urgence.
En second lieu, en présence d'un terrain construit en zone à risque, il importe que les règles de constructibilité soient durcies pour limiter autant que faire se peut les sinistres, que les risques encourus soient correctement et substantiellement couverts par l'assurance personnelle du propriétaire. En outre, lors de la cession du bien, une partie de la plus-value réalisée sera versée audit fonds. Les effets de la bulle financière ne sauraient bénéficier au seul propriétaire alors que maintes charges relèvent de la collectivité.

Il est peu probable que le droit de l'urbanisme permette d'atteindre de tels résultats en raison de sa complexité et du rapport qu'il entretient avec le droit de propriété privée. C'est plutôt du droit de l'environnement qu'il faut attendre de telles évolutions. Ceci est lié à la manière dont le droit de l'environnement s'est stratifié en France depuis 1970. En un premier temps, ce droit naissant a rassemblé diverses dispositions à l'encontre de l'exercice libéral du droit de propriété. De ce point de vue, il n'était pas très différent du droit du travail ou du droit de l'urbanisme. Dans un second temps, dans la décennie 1990, le droit de l'environnement est devenu une nouvelle branche du droit s'insinuant dans l'ensemble des domaines classifiés qui structurent le droit français. Il a trouvé dans ce cadre une force considérable et une logique de proposition. Enfin avec l'affirmation du concept de développement durable traduit en des normes juridiques, le droit de l'environnement est devenu une nouvelle forme de propositions et de structuration du droit de la propriété privée (Collard-Dutilleul et Romi, 1992).

Dans cet esprit, a été conçue la notion de «sols environnementaux » renvoyant aux secteurs sensibles et vulnérables. Pour ces espaces, le caractère absolu du droit de propriété pourrait être battu en brèche au motif que le sol constitue une ressource non renouvelable présentant un intérêt général. L'État pourrait exercer un «nouveau domaine universel », qui n'est pas sans rappeler la « directe » ou le «droit éminent» du droit français d'avant 1789. Le droit de propriété privé ne serait pas supprimé mais la chose sur laquelle porterait ce droit pourrait donner lieu à un ensemble de sujétions normalisées immédiatement opposables au propriétaire de la chose. Celui-ci posséderait un volant de droits réels et de devoirs, ce qui, en secteur sensible ou vulnérable, le placerait davantage en position de 
responsabilité. L'État serait en mesure de lui opposer ses propres droits supérieurs. La dissociation finance et droit sortirait clarifiée de cette distinction et le pouvoir régalien, que nous appelions de nos vœux, exercerait efficacement, y compris dans sa traduction administrative, les prérogatives de la souveraineté (Trébulle, 2006, 2010). Comme indiqué ci-dessus, pour ne pas brouiller la lisibilité des documents administratifs, le préfet de région devrait avoir la haute main sur les documents d'urbanisme de toutes les communes littorales non seulement pour réguler l'utilisation des sols mais également pour éviter que les espaces vulnérables soient urbanisés à mauvais escient.

\section{Propriété et mâ̂Trise DES « SOLS ENVIRONNEMENTAUX »}

Il faut savoir concilier respect de la nature et présence humaine. Dans cet esprit, il est raisonnable de concevoir que certains lieux ne soient pas habités ou le soient de façon très encadrée. Les notions de réserves naturelles, de sites protégés, de paysages remarquables, de parcs nationaux, de biens acquis par le Conservatoire du littoral, etc., sont désormais suffisamment familières et sont clairement définies dans notre législation qui en a créé le principe et a veillé à structurer leur organisation.

La notion de "sols environnementaux » justifie sans barguigner que certains espaces soient soustraits au marché immobilier. Nous avons suffisamment insisté dans le cadre de cet article sur la mécanique du droit de propriété couplée à la volonté de s'approprier certains biens en bord de mer, pour ne pas y revenir. La quête de l'appropriation est insatiable et il faut peu compter sur la capacité de régulation de la part des citoyens.

Dans chaque département, mais aussi à l'échelle régionale voire interrégionale afin d'être en présence d'une aire pertinente, la protection de certains secteurs s'impose. Ces lieux sensibles présentent un caractère de vulnérabilité. Certes les tenants de la deep ecology auraient bien voulu que ces lieux, comme certains arbres ou animaux, soient dotés de la personnalité juridique afin d'être défendus plus facilement, y compris devant des juridictions. Cette volonté n'a pas connu une telle traduction dans notre législation (Ost, 2003). Mais il est des lieux vulnérables qu'il faut protéger et des popula- tions qui ne doivent pas, pour des raisons de sécurité, s'installer en ces dits lieux. L'application des règles habituelles en matière d'urbanisme étant parfois aléatoire, mieux vaut adopter des politiques volontaristes. L'exemple de plusieurs espaces correctement cartographiés, notamment dans les communes inondées lors de la tempête Xynthia, montre qu'il aurait fallu en son temps les retirer du marché foncier. Nous avons également souligné ci-dessus que dans le Département de la Vendée, des coupures vertes ont pu être maintenues, y compris en des lieux situés en bordure de mer, bénéficiant de points de vue remarquables. Ces lieux appartiennent à l'État ou sont placés sous son étroit contrôle. Les géographes savent parfaitement ce qu'il est advenu des aires littorales que l'État a, voici de nombreuses années, cédé à des particuliers dans le cadre d'échanges.

La seule manière de parvenir à une parfaite mâ̂trise suppose que des parcelles soient identifiées et registrées comme lieux à protéger. La cartographie et la somme d'informations collectées dans chaque département le permet. Dans la panoplie qu'offre la loi, il est une procédure qui peut prendre plusieurs voies : la «domanialisation». L'État ou une collectivité autorisée peut exproprier pour cause d'utilité publique, peut se lancer dans une acquisition amiable ou bénéficier d'un droit de préemption lorsqu'il a été établi. Les ressources pour y parvenir pourraient être puisées dans le fonds créé par la taxation de la rente foncière. Dans le cas d'espèce, ou la politique est volontariste ou elle n'est que velléitaire.

Ensuite se pose la question de la gestion de ces espaces placés sous la directe de l'État. Jusqu'à présent, des solutions ont été trouvées, notamment avec le concours de l'Office National des Eaux et Forêts, le Conservatoire du littoral et l'aide efficiente des collectivités locales. Dans le secteur considéré, le tourisme s'accommode volontiers des coupures vertes, des réserves d'oiseaux, des zones dites naturelles, et bien entendu des paysages remarquables. Leur mise en valeur peut être conçue tout en restant respectueuse de la nature des lieux. Les solutions, dans le cadre d'une affectation, sont plurielles et contrôlables. 


\section{Conclusion}

Le droit de l'urbanisme prend appui sur le droit de propriété dont il n'infléchit que partiellement les dispositions, et ce sur un mode spécialisé. En une période où, après la tempête Xynthia, il est question de légiférer ou de renforcer la réglementation en cours, il ne faut pas oublier que ces textes nouveaux, une fois adoptés, pourront rester lettre morte ou de peu d'efficience si le droit de propriété n'est pas sensiblement modifié. Les perspectives qu'ouvre la notion de «sols environnementaux » méritent d'être explorées en ce qu'elles créent une directe de l'État sur l'ensemble du territoire français. Une telle disposition, qui s'inscrit en opposition avec la position adoptée par la jurisprudence française depuis la première moitié du XIX ${ }^{e}$ siècle, ouvrira la voie à des modes d'actions renouvelés au bénéfice d'un État qui retrouvera dans ses fonctions de protection et de contrôle, des prérogatives de souveraineté. Dans un tel cas de figure, il est possible de penser que le marché sera encadré et non laissé, comme les exemples récents l'ont tristement montré, au gré des sollicitations et des envies non réfrénées.

\section{Bibliographie}

BÉCET J.-M., 2002. Le droit de l'urbanisme littoral, Rennes, PUR, $249 \mathrm{p}$.

Bergel J.-L. (dir.), 2002. Les grand arrêts du droit immobilier, Paris, Dalloz, p. 3-118.

Chauvet A., Renard J., 1978. La Vendée, le pays, les hommes, Les Sables-d'Olonne, Le Cercle d'Or, p. 166-181.

Collard-Dutilleul F., Romi R., 1992. Propriété et protection de l'environnement, Rapport ministère de l'Environnement, $492 \mathrm{p}$.

Halbwachs M., 1908. La politique foncière des municipalités, Brochure de la Librairie du Parti Socialiste, coll. « Les cahiers du socialiste », $\mathrm{n}^{\circ} 3,31 \mathrm{p}$.
Hussenet J., 2004. Le tourisme littoral, Subir ou accueillir?, Des curés aux entrepreneurs, La Vendée au $\mathrm{xx}^{e}$ siècle, La Roche-sur-Yon, Centre vendéen de recherches historiques, p. 653-682.

Hussenet J., 2008. La côte vendéenne dans 20 ans, essai de prospective, Les Vendéens et la mer, de la grande pêche au Vendée Globe, La Roche-sur-Yon, Éditions du CVRH, p. 713-724.

Lebreton J.-P. (dir.), 2009. Évolution juridique des premiers schémas de cohérence territoriale, Les cahiers du GRIDAUH, Série Droit de l'urbanisme, $\mathrm{n}^{\circ} 19,255 \mathrm{p}$.

Legal P.-Y., 2008. L'urbanisation de Merlin-Plage à la loi littoral, Les Vendéens et la mer, de la grande pêche au Vendée Globe, La Roche-sur-Yon, Éditions du CVRH, p. 649-662.

LÉvy J.-Ph., Castaldo A., 2002. Histoire du droit civil, Paris, Dalloz, p. 540-546.

Maulin E., 2003. Vº Souveraineté, dans Alland D., Rials S., Dictionnaire de la culture juridique, Paris, Lamy/PUF, p. 1434-1439.

Mercier D., Acerra M. (dir.), 2011 . Xynthia, une tragédie prévisible, Nantes, Place Publique, 64 p.

Ost F., 2003. La nature hors la loi, l'écologie à l'éprewve du droit, Paris, La Découverte, p. 147-204.

Patault A.-M., 1989. Introduction historique au droit des biens, Paris, PUF, coll. «Droit fondamental », p. 220-225.

Renard J., 2008. L'impact du tourisme sur les évolutions des populations du littoral vendéen (1954-2007), Les Vendéens et la mer, de la grande pêche au Vendée Globe, La Roche-surYon, Éditions du CVRH, p. 663-686.

Roвin M., 2002. La télédétection, des satellites aux systèmes d'information géographiques, $2^{\mathrm{e}}$ éd., Paris, Nathan Université, coll. «Fac géographie », 318 p.

Terré F., Simler P., 2006. Les biens, Paris, Dalloz, p. 85-144.

Trébulle F.-G., 2006. Le régime des biens environnementaux : propriété publique et restriction administrative au droit de propriété, La propriété, Association Henri Capitant, vol. 53, Paris, Éditions SLC, 608 p.

Trébulle F.-G., 2010. L'environnement et le droit des biens, Le droit de l'environnement, Association Henri Capitant, Paris, Dalloz, $178 \mathrm{p}$.

Xifaras M., 2004. La propriété, Étude de philosophie du droit, Paris, PUF, coll. « Fondements de la politique », p. 479-504.

Zenati-Castaing F., Revet T., 2008, Les biens, Paris, PUF, coll. «Droit fondamental », 260 p. 